\title{
UNE-ISO 16175 SOBRE GESTIÓN DE DOCUMENTOS EN OFICINAS ELECTRÓNICAS: ALCANCE Y LIMITACIONES
}

\section{Vicent Giménez-Chornet}

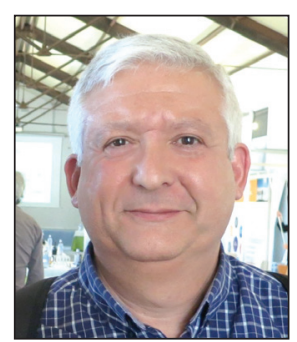

Vicent Giménez-Chornet es profesor titular del área de Documentación en la Universidad Politécnica de Valencia. Miembro del Comité 50 de Documentación de Aenor (Asociación Española de Normalización y Certificación). Director del máster oficial interuniversitario en gestión cultural. Ha sido presidente del Col/legi Oficial de Bibliotecaris i Documentalistes de la Comunitat Valenciana entre 2010 y 2012, secretario de Fesabid, archivero en el Archivo del Reino de Valencia, y presidente de Avei (Associació Valenciana d'Especialistes en Informació). http://orcid.org/0000-0003-1183-9058

Universidad Politécnica de Valencia Departamento de Comunicación Audiovisual, Documentación e Historia del Arte Camino de Vera, s/n. 46022 Valencia vigicho@har.upv.es

\section{Resumen}

Se analiza la norma UNE-ISO 16175 para determinar su interés y alcance en la gestión de los documentos electrónicos, permitiendo una mejor gestión de los documentos de archivo en las organizaciones. Se analizan también sus carencias o limites, que son generalmente resueltos por otras normas.

\section{Palabras clave}

Documentos de archivo, Gestión de documentos electrónicos, Sistema de gestión de documentos electrónicos, Requisitos para la gestión de documentos.

Title: UNE-ISO 16175 on document management in the paperless office: scope and limitations

\section{Abstract}

The UNE-ISO 16175 is analyzed to determine its scope and merits in the management of electronic records, enabling better archives management in organizations. There are analyzed its shortcomings or limitations that are generally resolved by other standards.

\section{Keywords}

Records, Archive documents, Digital records management, Systems, Requirements for record management.

Giménez-Chornet, Vicent (2013). “UNE-ISO 16175 sobre gestión de documentos en oficinas electrónicas: alcance y limitaciones". El profesional de la información, septiembre-octubre, v. 22, n. 5, pp. 455-458.

http://dx.doi.org/10.3145/epi.2013.sep.11

\section{Introducción}

Las normas ISO (International Organization for Standardization) son de voluntario cumplimiento, pero se usan porque las entidades encuentran beneficio al hacerlo (BusteloRuesta, 2012). Algunos países elaboran normas propias y otros dejan que sus entidades adopten estándares aprobados por organismos internacionales. Situándonos en España, si se quiere afrontar un buen modelo para la gestión de documentos electrónicos, se deben adoptar estándares generados por entidades internacionales, con la excepción del Esquema nacional de interoperabilidad (PAE) que se centra en los aspectos de interoperabilidad entre las administraciones.
Sobresalen dos estándares que establecen los requisitos funcionales que se deberían cumplir en la gestión de documentos producidos en la administración electrónica de cualquier entidad:

- Moreq (Modelo de requisitos para la gestión de documentos electrónicos), elaborado en 2001 en la Comisión Europea dentro del programa IDA (Interchange of data between administrations) e impulsado por la Fundación DLM Forum. Lleva dos actualizaciones generadas tras las sugerencias de mejora expresadas por sus usuarios, y su versión más reciente se ha retitulado Modular requirements for records systems 2010.

http://www.dlmforum.eu 


\section{http://moreq2010.eu}

- La norma 16175, publicada por ISO y editada en tres partes entre 2010 y 2011, que ha sido adoptada por Aenor como norma UNE-ISO 16175 en 2012, con el título Información y documentación. Principios y requisitos funcionales para documentos en entornos de oficina electrónica. Como la gestión de los documentos electrónicos sólo se puede realizar mediante un software (ya no sirven depósitos, estanterías, compactos, carpetas colgantes, cajas, señalética, fichas índice, fichas catalográficas, listados de cuadros de clasificación, listados de inventarios, etc.), cabe trasladar el modelo de gestión manual a unos requerimientos que debe cumplir el software para gestionar los documentos electrónicos producidos por organizaciones o personas en el desarrollo de su actividad, asegurando su integridad, autenticidad y validez a lo largo del tiempo, y que puedan ser recuperados cuando sea necesario.

\section{Alcance}

La norma UNE-ISO 16175 (2012) tiene tres partes que se venden separadamente.

\section{Generalidades y declaración de principios.}

2. Directrices y requisitos funcionales para sistemas que gestionan documentos electrónicos.

\section{Directrices y requisitos funcionales para documentos de la organización.}

Su objetivo principal es la elaboración de unas directrices y requisitos para la producción y gestión de documentos electrónicos. Directrices que debe observar la organización y requisitos que debe cumplir el sistema y el software correspondiente.

En la parte 1 se expone quiénes pueden ser los usuarios potenciales de esta norma, haciendo énfasis en que puede ser de utilidad a:

- responsables de crear un software para la gestión de los documentos electrónicos de archivo;

- organismos gubernamentales, en cuanto les puede permitir tener un modelo para evaluar las prestaciones automatizadas ya implantadas en la gestión de los documentos;

- sector privado, para poder incorporar un modelo de gestión de los documentos electrónicos en sus procesos de negocio.

Trata también de las buenas prácticas cuando las organizaciones deciden introducir las nuevas tecnologías para gestión y control de los documentos. Resalta unos principios rectores que deben considerar las organizaciones que necesiten un sistema de información donde la creación, conservación y uso de los documentos sea la evidencia o prueba de su actividad y satisfaga las necesidades de negocio. Entre ellos se indica, por ejemplo, que la información electrónica tiene que ser gestionada de forma activa y conservada de modo fiable como evidencia auténtica - prueba o testimonio en la bibliografía clásica de la archivística española- de la actividad, o que la información de la organización debe estar vinculada a su contexto mediante el uso de metadatos, etc.
Se plantean aspectos sobre la implementación, ya que un software de calidad es sólo un componente en la gestión de los documentos electrónicos de la organización, y señala otros componentes como el marco de políticas, tanto del contexto legislativo como las políticas de la organización, la gestión de proyectos o la gestión del cambio. Asimismo advierte de algunos riesgos que se deben considerar a la hora de seleccionar el software que se implementará en la gestión de los documentos, finalizando con algunos aspectos sobre sostenibilidad financiera, en definitiva una inversión de capital acorde a la estrategia de negocio.

La parte 2 se centra en dos aspectos:

- determinar las directrices para un sistema que gestiona los documentos electrónicos (capítulo 4);

- establecer los requisitos funcionales que debe soportar el software en ese sistema (capítulo 5).

Entre las directrices se señalan los aspectos esenciales que se deben tener en cuenta:

- para que los atributos de los documentos de archivo (autenticidad, fiabilidad, integridad y usabilidad) se mantengan efectivos, para hacer posible la producción de los documentos en su contexto, mantenerlos en el tiempo exigido y poderlos recuperar por sus metadatos;

- riesgos en los que podría caer la organización si no se implementa un sistema de gestión de documentos electrónicos (como incumplir requisitos legales, desacreditación de la dirección ejecutiva si se demuestra su incapacidad de gestionar la información de la organización de manera competente, etc.);

- se señalan los beneficios si implementa el sistema de gestión (protección de los intereses de la organización, capacidad de prestar servicios más eficiente, etc.).

Los requisitos funcionales son un conjunto de requerimientos demandados al software, con dos niveles de exigencia (el "debe", obligatorio, y el "debería", aconsejable y a valorar por la organización) para que la gestión de documentos que se realizaba en la archivística tradicional de forma manual ahora se pueda cumplir en la gestión electrónica. Entre ellos están la captura (o el ingreso) de los documentos en el sistema, la vinculación a su contexto, la identificación, la clasificación, los niveles de clasificación, el control del acceso, los controles de seguridad, los metadatos de la gestión -incluidos los referidos a la disposición de los documentos reflejados en las Tablas de valoración o retención documental- la trazabilidad, las transferencias, los sistemas de búsqueda o recuperación de la información, etc. Algunos de estos requisitos funcionales del capítulo 5 son coincidentes con el Moreq europeo.

La parte 3 se centra también en dos aspectos desde la perspectiva de la organización:

- directrices que ésta debe contemplar (capítulo 2);

- sus requisitos funcionales (capítulo 3).

El objetivo de esta tercera parte es comprender los procesos y requisitos para identificar y gestionar los documentos en los sistemas de la organización y desarrollar los requisitos de la función documental. La norma podrá servir a la orga- 
nización para revisar esa función documental o evaluar el grado de cumplimiento de los sistemas ya existentes. Los destinatarios de esta norma son principalmente el personal responsable del diseño, revisión y/o implementación de sistemas en la organización (analistas de negocio, responsables en las adquisiciones de TIC), los profesionales de los documentos que asesoran en los procesos, y los proveedores de software y desarrolladores que deseen incorporar funciones concretas para la gestión de los documentos electrónicos.

Las directrices resaltan la importancia de la gestión de los documentos, y proponen asuntos y procesos que debe considerar la organización a la hora de revisar, diseñar o adquirir funciones para su sistema de gestión. Entre las directrices trata la relevancia de conservar las evidencias de los procesos y actividades de la organización, de la integración de la gestión de documentos en las bases de procesos definidos (flujos de trabajo), y propone una serie de pasos para determinar los requisitos en la gestión de los documentos:

- análisis del proceso de trabajo;

- identificación de los requisitos de las evidencias;

- identificación del contenido y la información asociada a la evidencia, sus vínculos y dependencias (como localización, tamaño, formatos de archivo, seguridad, protección de datos, pistas de auditoría, etc.);

- diseño de estrategias para gestionar los procesos documentales que se consideran esenciales para la organización (por ejemplo, su contenido debe ser inalterable, para considerarlo auténtico y fiable);

- evaluación de riesgos como estrategia adecuada de la organización;

- propuesta e implementación (considerando los roles y las funciones de los usuarios) de un plan que deba ser tratado por equipos del proyecto (formado por expertos externos, propietarios del negocio, profesionales archiveros, expertos en riesgos, etc.) para el desarrollo de una especificación en el diseño del sistema de la organización.

Los requisitos funcionales están sometidos a tres niveles de exigencia

- el "debe", de imprescindible cumplimiento;

- el "debería", se debe sopesar su grado de cumplimiento;

- el "puede", opcional.

Los requisitos son 125 y se estructuran en cuatro apartados:

- creación de los documentos en su contexto: documento fijo con los metadatos del proceso de negocio y el contexto de la organización;

- configuración de los metadatos: debe permitir, entre otras funciones, que el administrador del sistema defina la fuente de datos de cada elemento de los metadatos durante la configuración del sistema, o debería dar soporte al movimiento de los documentos proporcionando mecanismos para la reasignación y reclasificación de los documentos dentro del sistema de la organización);

- soporte a la importación, exportación e interoperabilidad, como importación de la información de las pistas de autoría asociadas a documentos, o la exportación de documentos dentro del sistema de la organización o a una institución archivística que tenga un programa de conservación de documentos a largo plazo;
- conservación/retención de los documentos según la disposición establecida en las Tablas de valoración/calendarios de conservación dictadas por órganos autorizados.

\section{Limitaciones}

Las normas no abarcan, en general, toda la actividad de una entidad, sino que proponen regular algunos procesos concretos que pueden beneficiarse de una buena práctica. En algunos casos estos procesos pueden ser certificables. La gestión de los documentos electrónicos, nativos o incorporados al sistema desde otros formatos, contempla muchas facetas que son tratadas por varios estándares. La UNE-ISO 16175 ya indica en algunos casos sus límites, aquello que no aborda, y de lo que el usuario podrá encontrar mejor información en otras normas. Algunas limitaciones son:

- Regular la incorporación al sistema de documentos nacidos en papel mediante la digitalización. Las normas UNEISO sobre digitalización son la UNE-ISO/TR 13028:2011 IN Información y documentación. Directrices para la implementación de la digitalización de documentos, y la UNEISO/TR 15801:2008 IN Imagen electrónica. Información almacenada electrónicamente. Recomendaciones sobre veracidad y fiabilidad.

- Regular el empaquetado de la información, que conserve los metadatos de los documentos electrónicos, para su conservación y difusión. Es el modelo Open archival information system (OAIS) que ha sido adoptado como norma ISO 14721:2012 Space data and information transfer systems. Open archival information system (OAIS). Reference model, y la ISO 20652:2006 Space data and information transfer systems. Producer-archive interface. Methodology abstract standard.

- Regular el intercambio de copias de documentos electrónicos entre sistemas informáticos, tratado extensamente en la UNE-ISO 17933:2006 GEDI. Intercambio de documentos electrónicos genérico (ISO 17933:2000).

- Regular la calidad en el proceso, tratado en las normas UNE-EN ISO 9000:2005 Sistemas de gestión de la calidad. Fundamentos y vocabulario (ISO 9000:2005); UNE-EN ISO 9001:2008/AC:2009 Sistemas de gestión de la calidad. Requisitos (ISO 9001:2008/Cor 1:2009); y UNE-EN ISO 9004:2009 Gestión para el éxito sostenido de una organización. Enfoque de gestión de la calidad (ISO 9004:2009).

- Regular el formato de fichero para la conservación de los documentos a largo plazo. Una propuesta es el formato pdf: UNE-ISO 19005-1:2008 Gestión de documentos. Formato de fichero de documento electrónico para la conservación a largo plazo. Parte 1: Uso del PDF 1.4 (PDF/A-1).

- Regular el proceso de valoración y selección de documentos. Actualmente no existe ninguna norma, y algunos países regulan vía legislación cómo establecer dicho proceso para que queden reflejados en unas Tablas de valoración / Tablas de retención / Calendarios de conservación para los documentos generados en las administraciones públicas, y que tengan validez jurídica los documentos que se ha aprobado destruir.

- Regular un diseño básico de recuperación de la información. Actualmente se ha avanzado en la normativa relacio- 
nada con la recuperación mediante tesauros: ISO 25964 1:2011 Information and documentation. Thesauri and interoperability with other vocabularies. Part 1: Thesauri for information retrieval; ISO 25964-2:2013 Information and documentation. Thesauri and interoperability with other vocabularies. Part 2: Interoperability with other vocabularies

- Se complementa con el análisis de los procesos de trabajo desde el punto de vista de la creación, incorporación y control de documentos, tratado en la norma UNE-ISO/TR 26122:2008 IN Información y documentación. Análisis de los procesos de trabajo para la gestión de documentos.

- Se complementa con la extensa norma que trata de los metadatos para la gestión de documentos, publicada en tres partes: UNE-ISO 23081 Información y documentación. Procesos de gestión de documentos. Metadatos para la gestión de documentos, Parte 1 (2008), Parte 2 (2011), y Parte 3 (2012).

- Se complementa con los criterios generales de las estrategias a seguir para la conservación de la información que ha quedado registrada en los documentos, establecidas en la UNE-ISO/TR 18492:2008 IN Conservación a largo plazo de la información basada en documentos.

- Se complementa con la norma que ofrece la metodología para un enfoque sistemático en la creación y gestión de documentos, alineada con los objetivos y estrategias de la organización, proponiendo criterios que debe seguir una organización para la buena gestión de los documentos: UNE-ISO 30300:2011 Información y documentación. Sistemas de gestión para los documentos, UNE-ISO 30301:2011 Información y documentación. Sistemas de gestión para los documentos. Requisitos. Pronto se publicarán las normas complementarias: 30302 sobre la guía de implementación, 30303 sobre requisitos para los organismos que realicen la auditoría y la certificación, y 30304 sobre guía de evaluación.

- Se complementa con la norma que trata de la gestión de los documentos de archivo: UNE-ISO 15489-1:2006 Información y documentación. Gestión de documentos. Parte 1: Generalidades, y UNE-ISO/TR 15489-2:2006 Información y documentación. Gestión de documentos. Parte 2: Directrices (ISO/TR 15489-2:2001).

\section{Conclusión}

La norma UNE-ISO 16175 establece en sus tres partes los principios de buenas prácticas mediante una propuesta de directrices que deben cumplir el sistema y la organización, y una propuesta de requisitos funcionales que debe cumplir el software que controlará el sistema de gestión de los documentos en un entorno de oficina electrónica. La norma UNE-ISO 16175 es más amplia que el Moreq, ya que éste se limita a los requerimientos funcionales del sistema o software, mientras que la norma abarca también las directrices que debe cumplir la organización para el correcto funcionamiento de la oficina electrónica y garantizar la gestión de unos documentos auténticos, íntegros y fiables. Otras normas pueden resolver las limitaciones de la UNE-ISO 16175 en las diversas actividades que se puedan plantear en una entidad.

La norma UNE-ISO 16175 es más amplia que el Moreq, ya que éste se limita a los requerimientos funcionales del sistema o software

\section{Bibliografía}

Bustelo-Ruesta, Carlota (2012). "La normalización internacional en información y documentación: ¿una historia de éxitos? El caso de la normalización ISO en gestión de documentos". Métodos de información (MEI), II época, v. 3, n, 4, pp. 39-46.

http://dx.doi.org/10.5557/IIMEI2-N2-039046

DLM Forum (2013). About MoReq2010. Modular requirements for records systems. DLM Forum Fundation.

PAE, Portal de Administración Electrónica. Esquema Nacional de Interoperabilidad.

http://administracionelectronica.gob.es/ctt/ verPestanaGeneral.htm?idIniciativa=eni

UNE-ISO 16175-1:2012 (2012). Información y documentación. Principios y requisitos funcionales para documentos en entornos de oficina electrónica. Parte 1: Generalidades y declaración de principios. Madrid: Aenor.

UNE-ISO 16175-2:2012 (2012). Información y documentación. Principios y requisitos funcionales para documentos en entornos de oficina electrónica. Parte 2: Directrices y requisitos funcionales para sistemas que gestionan documentos electrónicos. Madrid: Aenor.

UNE-ISO 16175-3:2012 (2012). Información y documentación. Principios y requisitos funcionales para documentos en entornos de oficina electrónica. Parte 3: Directrices y requisitos funcionales para documentos en los sistemas de la organización. Madrid: Aenor. 